\title{
TRUEDOP - A NEW QUALITY STEP FOR OFFICIAL ORTHOPHOTOS
}

\author{
S. Baltrusch ${ }^{\mathrm{a}}$ \\ ${ }^{\text {a }}$ LAiV M-V, NMA - Photogrammetry Department, 19059 Schwerin, Germany - sven.baltrusch@laiv-mv.de
}

SpS 12 - EuroSDR: Innovative technologies and methodologies for NMCAs

KEY WORDS: ATKIS-DOP, Aerial Survey, TrueDOP, NMA, Dense Image Matching, image-based DSM

\begin{abstract}
:
The National Mapping Agencies (NMA) of the German federal states derive nationwide digital orthophotos with a ground resolution of 10 to $20 \mathrm{~cm}$ by aerial surveys at regular cycles of two or three years. Standard surveys use large format cameras, the direct georeferencing informations and normal overlap ratios of $70 \%$ forward and 30\% sideward overlap. These basic geodatasets are in use in a variety of applications. Most commonly authoritive agencies use these orthophotos as a fundamental dataset in their GISsystems.

Interactive work for deriving the orthopohotos is necessary for updating the terrain model and for defining seamlines for objects above the reference plane. With respect to the developments in dense image matching using the semi global matching algorithms it is possible to derive surface models with pixel resolution and full color informations of the aerial photos. Using these high resolutioned height informations for the orthophoto procedure some software solutions are able to derive the quality of true orthophotos without remaining occluded areas. The rectification additionally uses always a height model from the same survey so that there will be no interactive steps in the working process left. TrueDOP visualises the correct position of all objects without the fault effect of the central perspective. This is a basic condition for using the dataset for effective rasterbased classification applications, in special for the use in change detection.

The Working Committee of the Surveying Authorities of the States of the Federal Republic Germany (AdV) evaluates the replacement of the classical ATKIS-DOP by the TrueDOP. In this connection the TrueDOP is understood as a qualitative upgrading of the existing AdV-product ATKIS-DOP. The resulting advantages and disadvantages in reference to the technical and economical aspects are considered and compared.
\end{abstract}

\section{INTRODUCTION}

Digital Orthophotos (DOP) are raster datasets of the photographic presentation of the earth surface, which are principally free of distortion and true to scale. They are derived from orientated aerial photos and a digital terrain model. DOPs are based on photographs, which are raster-oriented, geocoded and provide precise positioning. They are not generalised, complete and provide a view with respect to their use.

The German National Mapping Agencies (NMA) realise cyclical aerial survey campaigns for their authorities by tender and derive DOP. According to a nationally standard of the AdV these DOPs are managed in the Topographic-Informationsystem ATKIS ${ }^{\circledR}$. DOPs are available with the ground resolutions of 20 $\mathrm{cm}$ (DOP20) and $40 \mathrm{~cm}$ (DOP40), in some German Länder also with the ground resolution of $10 \mathrm{~cm}$ (DOP10). The product specifications for DOP are specified in the product standard for digital orthophotos. The product standard is provided as a nocharge download (www.adv-online.de).

True Orthophotos are mainly characterized by the fact that occluded areas are removed and no tilting of objects above ground level is remaining. For the derivation of TrueDOP a high resoluted surface model is necessary.

The AdV evaluates the replacement of the classical ATKISDOP by the TrueDOP. In this connection the TrueDOP is understood as a qualitative upgrading of the existing AdVproduct ATKIS-DOP. Therefore studies were made with reference to the necessary technic modifications, organisationchanges in aerial surveys and postprocessing as well as in budget requests.

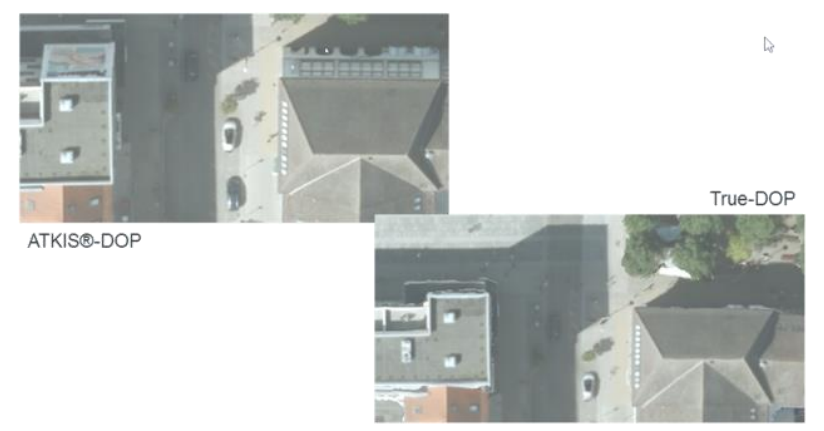

Figure 1. Left: ATKIS-DOP; Right: TrueDOP

Since the introduction of the Dense-Image-Matching (DIM)technology efficient production workflows of TrueDOP became realistic. DIM-technologies derive surface point clouds up to pixel-wide resolutions and can also combine these datasets with spectral informations. The color-coded surface informations reduced to a $2 \mathrm{D}$-space can be interpretated as a TrueDOP. Afterwards modern photogrammetric software solutions enforce radiometric improvements and geometric corrections, like edge adjustments. Contemporary the German NMAs have made experiences with TrueDOP-products derived from the software Surface Recognition SURE (nFrames GmbH). Meanwhile the processing of multispectral images (RGBI) is solved in these software. According to Trimble Inpho an implementation of 
similar workflows in their product chain (Match-T DSM and OrthoBox) is announced for the Intergeo 2016 in Hamburg.

The tilting of objects above ground level grows to the edges of aerial images caused by the central perspective projection. This effect is eliminated at TrueDOP orthorectification processes due to highly accurated and high-resolutioned digital surface models (DSM). Thus the derivation of TrueDOP is independent of additional digital height model informations.

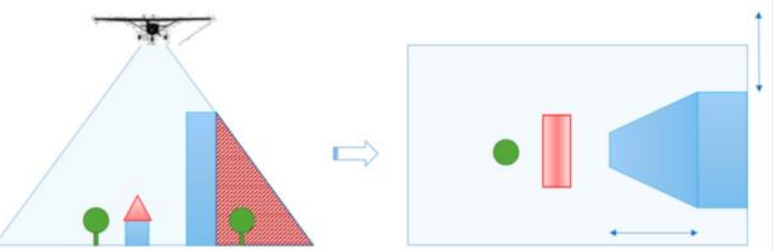

Figure 2. Tilting effects in classic DOP (Source: Wenzel, nFrames $\mathrm{GmbH})$

\section{TRUEDOP - ISSUES}

\subsection{Requirements to aerial survey parameters}

The TrueDOP can principly be obtained based on the same aerial image data from which even a classic ATKIS DOP has been derived, which has been prepared for the orthorectification on a ATKIS DGM. By TrueDOP algorithms tilting effects can be prevented and all objects on the ground can be displayed at the correct position. Occluded areas are filled by information from other perspectives. Optimized image flight parameters, in particular by increasing the forward and sideward overlapping, improve the image content by additional information derived from further evaluable angles, so that hidden areas can be minimized.

\begin{tabular}{|cc|}
\hline Forward overlap & Stereo Models \\
\hline$>\mathbf{5 0} \%$ & 2 \\
\hline$>67 \%$ & 3 \\
\hline$>\mathbf{7 5} \%$ & 4 \\
\hline$>\mathbf{8 0} \%$ & 5 \\
\hline
\end{tabular}

Table 1. Stereo models depending on Forward Overlaps

An optimal TrueDOP requires a gapless surface model with a reliable height accuracy. Depending on the locality and especially on the small-scale varieties of object-heights (buildings, narrow streets, bridges or even forest pathes) this can be achieved by increasing forward- und sideward overlapping parameters. Areas, where 2.5D-pixels cannot be derived, are figured out as "no-data-areas". If there is a high overlap the pixelinformations of nearby pixels can be used for color interpolation and afterwards smoothing.

a)

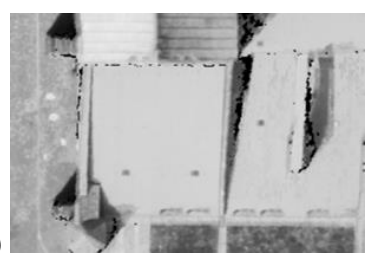

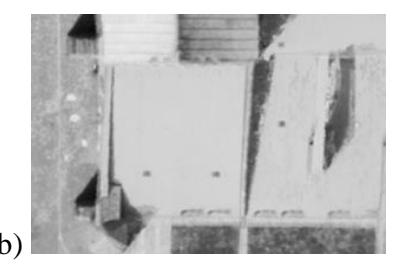

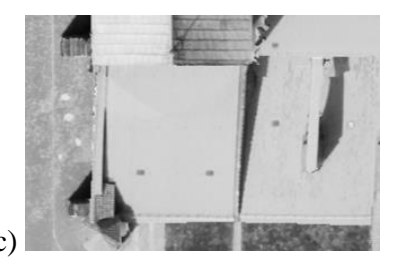

d)
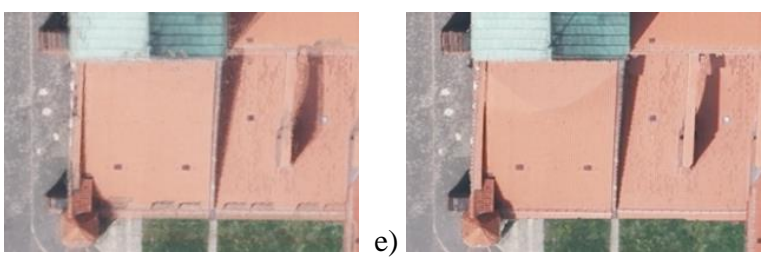

Figure 3. TrueDOP:

a) PAN- without postprocessing

b) PAN-with interpolation c) PAN-with antialiasing

d) RGBI-with interpolation e) RGBI-with antialiasing

Own experiences as well as statements of the software manufacturers prove that especially a forward overlap of $80 \%$ leads to a significant improvement of the image quality of the TrueDOP.
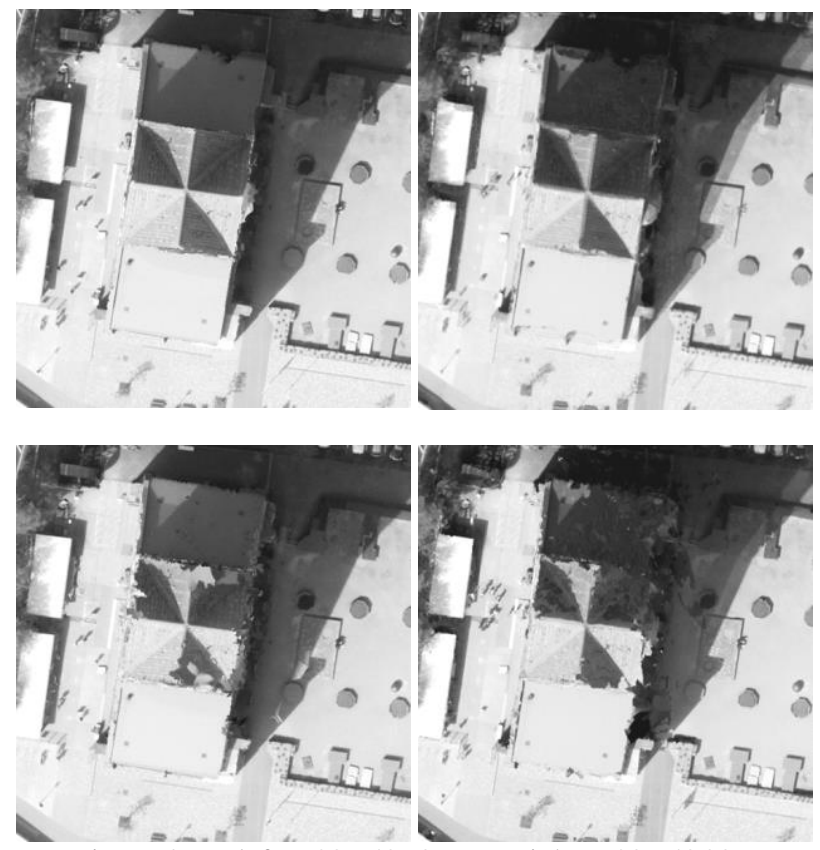

Figure 4. up-left: L80\%/Q60\%; up-right: L80\%/Q30\%;

down-left: L60\%/Q60\%; down-right: L60\%/Q30\%

On the other hand an exclusively increasing of the sideward overlap leads to less advantages, because the main correlation of the software is done in the images in flight direction (with homogenous lighting conditions). Increasing the sideward overlap can help to fill gaps across to the flight direction.

In terms of TrueDOP processing out of aerial survey projects of NMAs it is recommended to use forward overlapping $\mathrm{L}=80 \%$ and at least a sideward overlapping $\mathrm{Q}=30 \%$. These flight parameters are standard for the majority of NMAs aerial survey projects.

In dense urban areas, a covering of $\mathrm{L}=80 \%$ and $\mathrm{Q}=60 \%$ is recommended. Both parameter-sets (L80/Q60 and L80/Q30) can be well aeronautically combined within one aerial survey project. 


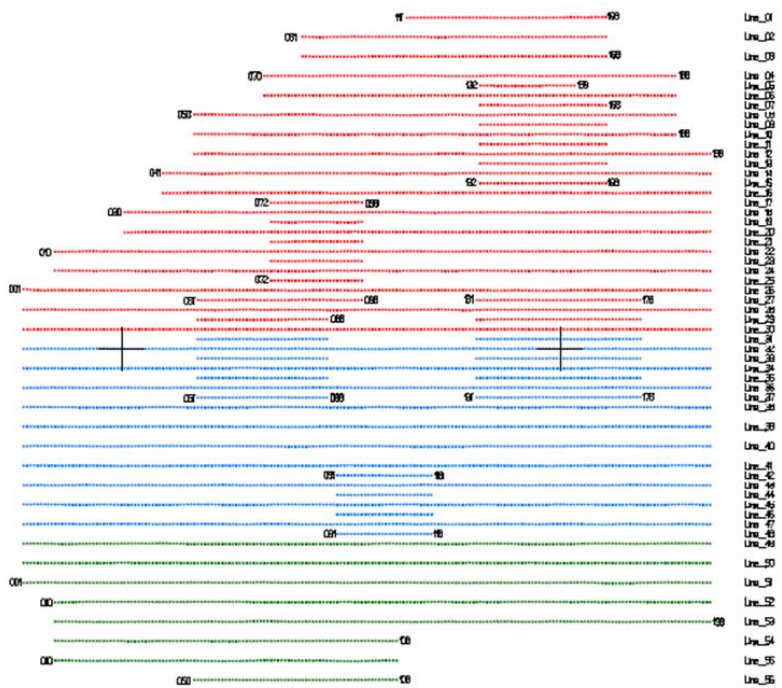

Figure 5. Aerial survey project - Q60\% and Q30\% combination The other aerial survey parameters behave identically to the parameters of the classic DOP workflow.

\subsection{Requirements to Hardware-environment}

Deriving TrueDOP is - similar to the production of image-based surface models - a very compute- and memory-intensive process. Therefore a high-performance and stable hardware environment is required. Current benchmarks have yet to be completed. The following factors influence the calculation time:

- Use of CPU or GPU processors

- Available hardware memory

- $\quad$ Server / Workstation / Network environment

- Memory System (S-ATA / Raid)

In the German Länder Bavaria and Mecklenburg-Vorpommern the following calculation times are measured:

\begin{tabular}{ccc}
\hline BY & GSD 20cm / L80\% / Q50\% & Ca. $30 \mathrm{~min} / \mathrm{km}^{2}$ for bDOM20 and TrueDOP20 \\
\hline MV & GSD10 $\mathrm{cm} / \mathrm{L80 \%} / \mathrm{Q} 30 \%$ & $1 \mathrm{~h} / \mathrm{km}^{2}$ for bDOM10 and TrueDOP10 \\
\hline
\end{tabular}

Table 2. Computing times for TrueDOP derivation

\subsection{Possible errors in TrueDOP}

A correct digital surface model with geometrically identical resolution is the precondition for a geometrically correct TrueDOP. Height errors in the surface model have a direct effect on the pixel representation from the digital aerial image into the TrueDOP.

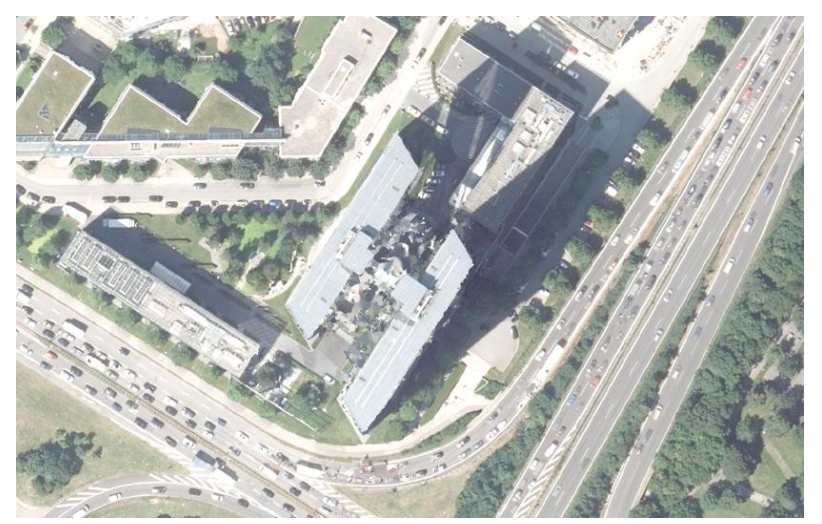

Figure 6. DSM-Error caused by occluded areas

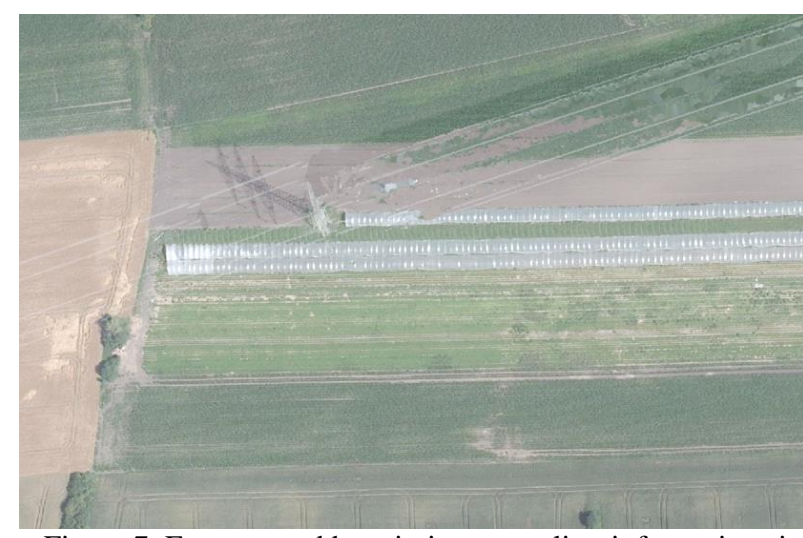

Figure 7. Error caused by missing powerline-informations in DSM

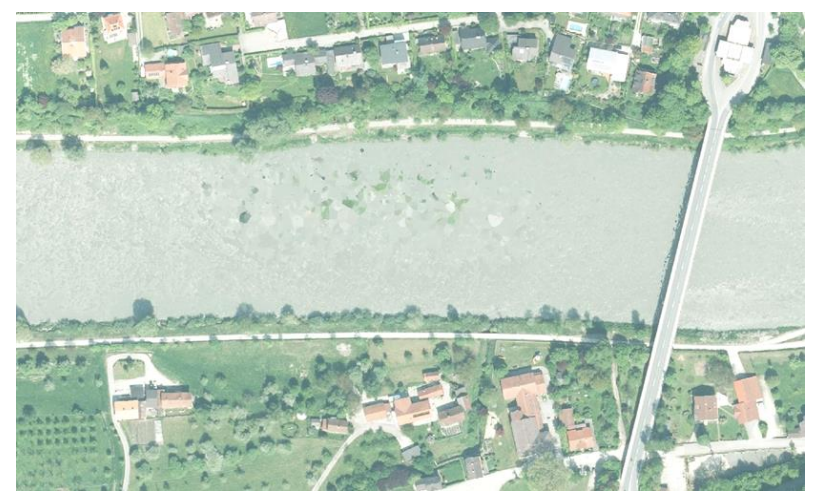

Figure 8. Error caused by DSM-noise in water areas

The above figures show that the manner of representation of the TrueDOP directly depends on the quality of the surface model. The following aspects of quality therefore have to be observed in the generation of the surface model and also have to be corrected interactively:

- Remove of position- and height offsets

- Remove of noise in the water surfaces

- Treatment of gaps in surface models

- Detect and eliminate outliers (for example, moving objects)

- Improve the corners of buildings (if necessary)

- Remove of altitude errors in DSM caused by faults in the aerial images (for example clouds or cloud shadows)

The potential of image-based surface models is not limited by the derivation of TrueDOP. The application potential extends currently also to the generation and updating of the Digital Terrain Model ATKIS-DTM (restricted), digital surface models DSM and the AdV-3D building models. Considering the varied fields of applications the effort to improve the quality of the DSM is justifiable.

\subsection{Advantages of TrueDOP in comparison to classical ATKIS-DOP}

TrueDOP show a lot of advantages in comparison to classical ATKIS-DOP:

- Correct mapping position of objects above ground level

- Improved results for image-based classification processes

- Conflict-free combination with other geodatasets

- Improved initial datasets for other georeferenced applications 
- $\quad$ Optimized mapping of bridges

- Increased positional accuracy

- No time difference between DOM and image recording

- Increased information content

The advantages have an effect on the geometrical accuracy, the dense of information as well as on the effort of human interactive working steps. Increasing quality in combination with reducing interaction are basic reasons for introducing TrueDOP in the ATKIS-production-chain.

\subsubsection{Correct mapping position of objects above ground} level: In classic orthophotos the representation of objects above ground level and the consequent tilting depends on the distance to the image center. Variations in the used digital camera sensors and the associated record areas combined with the project parameters $\mathrm{L}$ and $\mathrm{Q}$ lead to random tilting of objects.
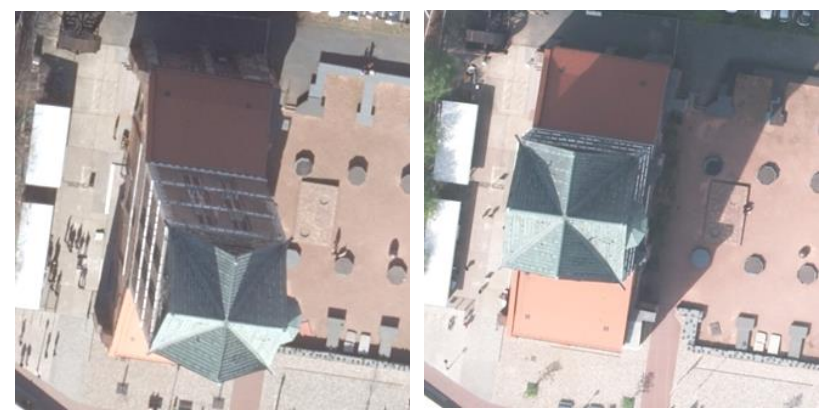

Figure 9. ATKIS-DOP (2014)

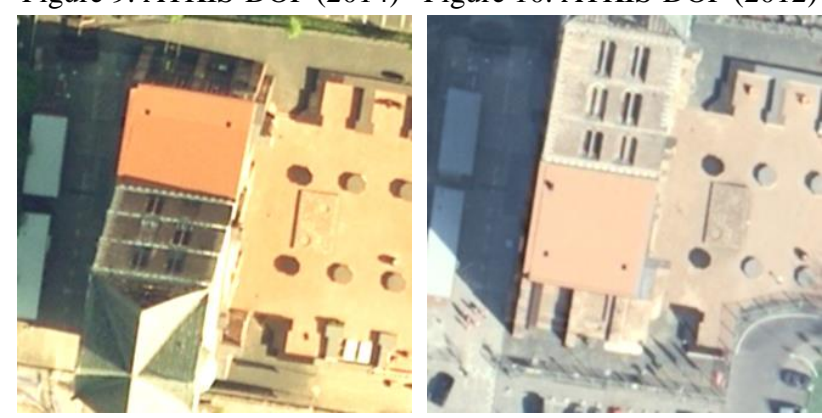

Figure 11. ATKIS-DOP (2008) Figure 12. ATKIS-DOP (2005)

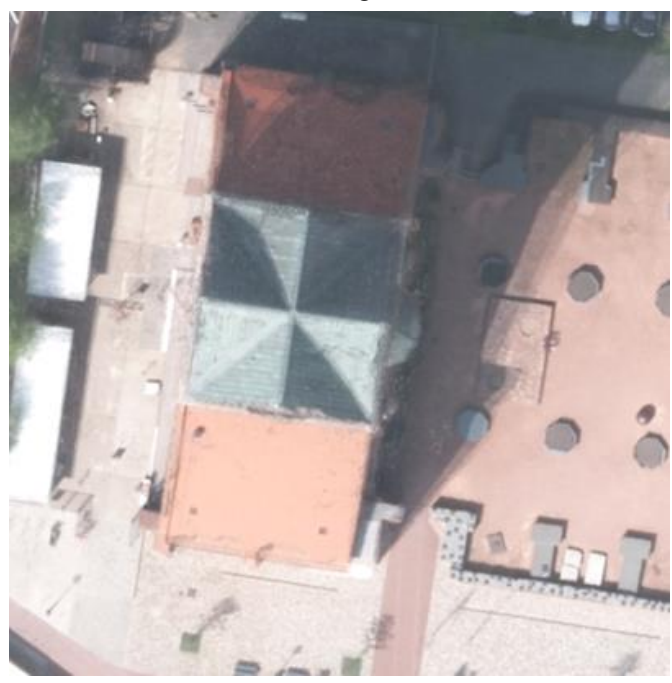

Figure 13. True-DOP (2012)

In consequence of the location-correct mapping of objects no seamlines in orthophoto mosaics are necessary. This leads to savings on interactive human efforts.
2.4.2 Improved results for image-based classification processes: In automated image-based classification methods such as Change Detection better results can be achieved due to the correct position of objects above ground level. Tilting caused error effects are omitted. Buildings can be classified at their correct position and can therefore be used for change analysis.

2.4.3 Conflict-free combination with other geodatasets: In contrast to the classical DOP in TrueDOP correct mapped objects such as buildings can be represented with other spatial data sets such as ALKIS / ALK without conflicts.

2.4.4 Improved initial datasets for other georeferenced applications: For other applications, such as forest-mapping, arise improved initial datasets for classifications or other georeferenced issues. The treetops are mapped at their correct position in TrueDOP. This is a requirement of the environmental and agricultural management.

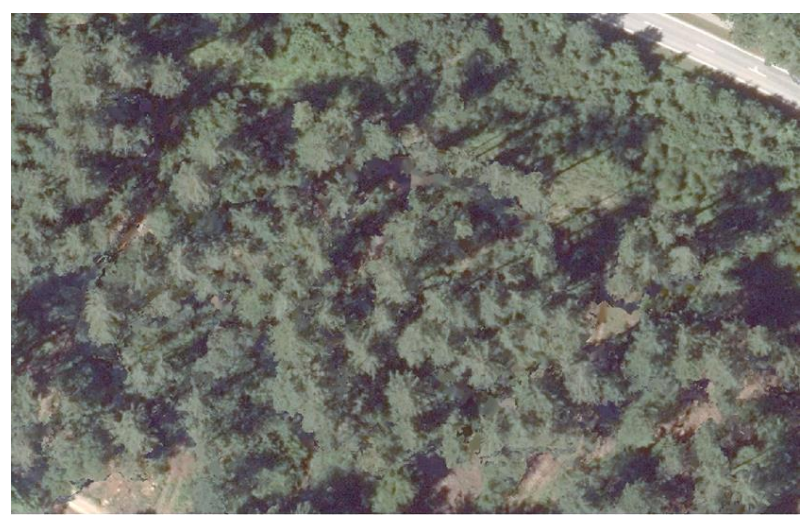

Figure 14. Forest in TrueDOP

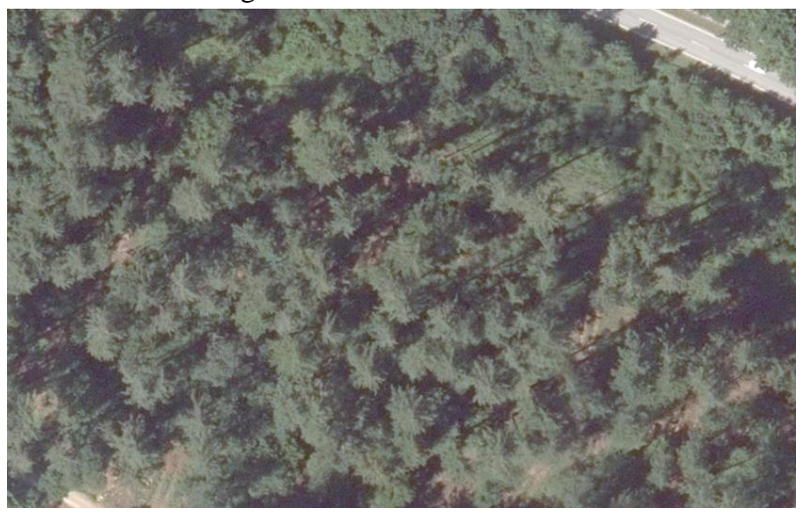

Figure 15. Forest in ATKIS-DOP

2.4.5 Optimized mapping of bridges: In classical ATKISDOP workflows particularly the topographical representation of bridge elements is a major challenge and represents a high interactive effort. The reason for this is that bridges by definition are not part of the DTM and therefore have to be modeled manually in the production process of ATKIS-DOP. In TrueDOP the bridge elements are automatically displayed correctly because bridges are integrated in the DSM. 


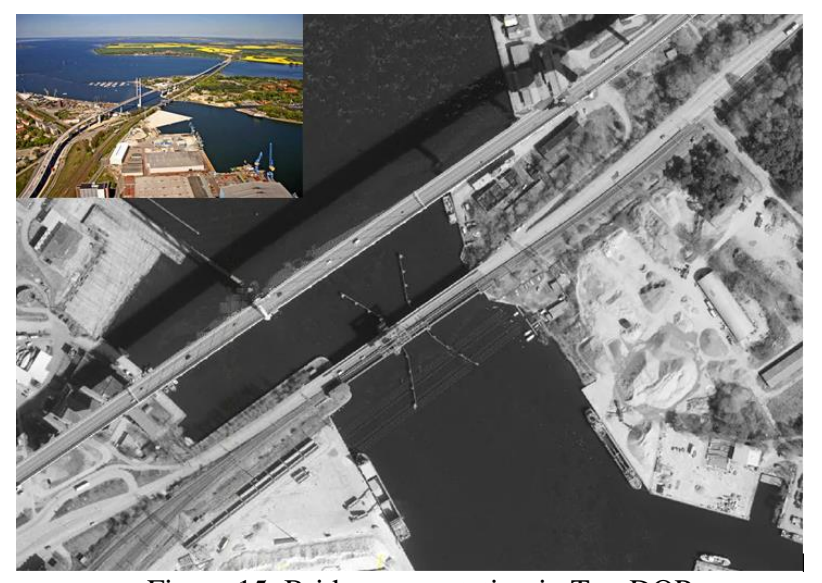

Figure 15. Bridge presentation in TrueDOP

2.4.6 Increased positional accuracy: Due to the correct position of objects (without tilting) object points (above the terrain model) can be digitized with the identical accuracy as ground points in TrueDOP. The uncertainty due to the tilted display is deleted.

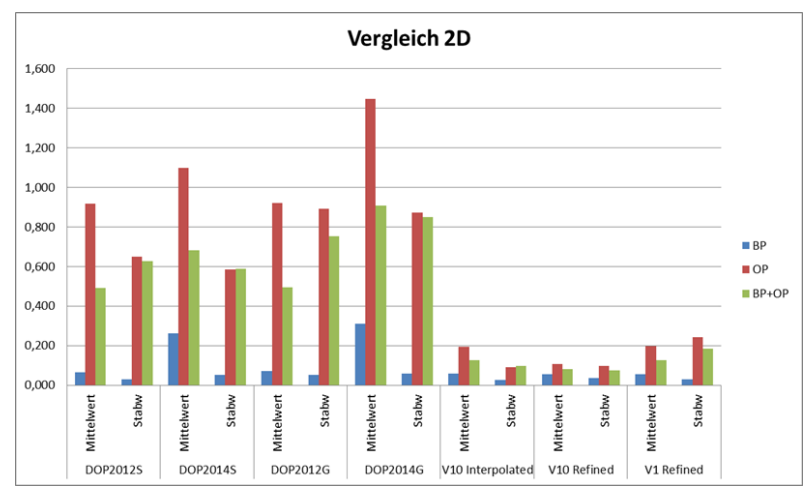

Figure 16. Comparison of 2D-accuracy for Ground Points (BP) and Object Points (OP)

2.4.7 No time difference between DOM and image recording: The quality of ATKIS-DOP is as well influenced by the orientation accuracy of the aerial images as on the used digital terrain model (DTM). DTM errors make an impact on the sitional error in ATKIS DOP with up to 50\%. Inaccuracies and possible missing actuality lead to positional errors in the orthophotos. In particular, linear elements such as railways or roads require interactive reworking.

This error source is not applied in TrueDOP processes, because the resolution of the initial DSM is pixel-sharp and the currentness of the DSM is identical to the capture-date of the aerial images.

2.4.8 Increased information content: Tilting leads to missing informations for occluded areas. By TrueDOP processes and corresponding image flight parameters, these areas can be filled and displayed as additional content in the TrueDOP from other viewing directions, so that the information content of TrueDOP compared to the classic digital orthophotos can be increased.

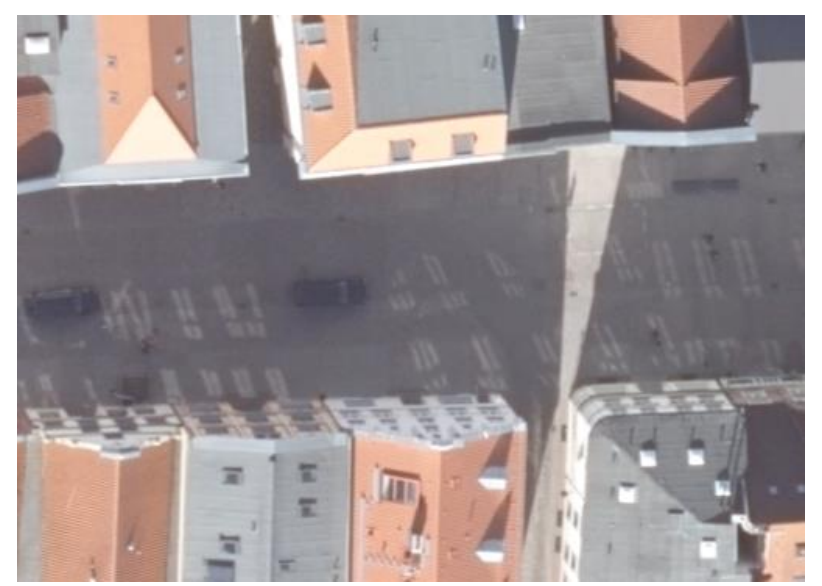

Figure 17. ATKIS-DOP with occluded areas in small alleys and backyards

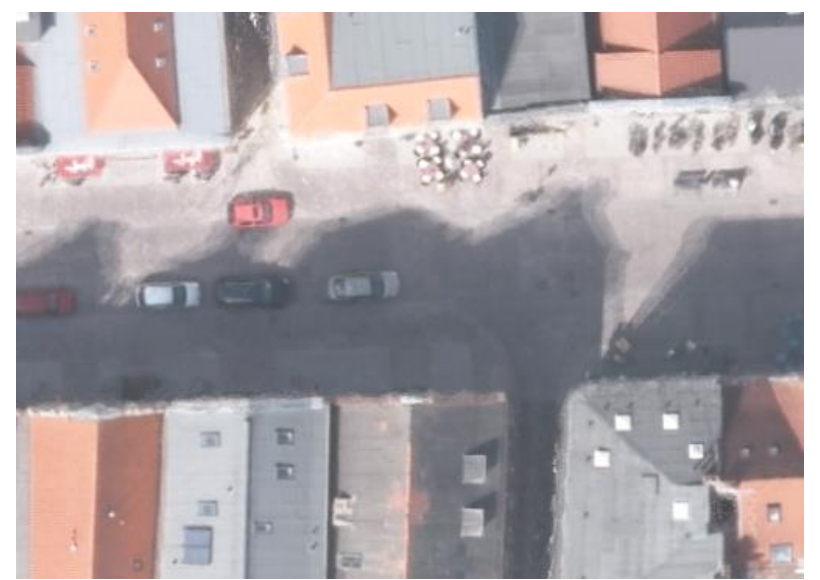

Figure 18. TrueDOP presentation without occluded areas

\subsection{Disadvantages of TrueDOP in comparison to classical ATKIS-DOP}

An honest comparison of the new technology and the classic production workflow concurrently shows some disadvantages, which also have to be figured out:

- Omission of height interpretation from perspective presentations

- Interactive effort shifted in the DSM-quality analysis

- Ghosting effects of mobile objects

- Remaining fringes at object-edges due to geometric and radiometric shadow

2.5.1 Omission of height interpretation from perspective presentations: In classic ATKIS-DOP users were able to interpretate relative height informations out of the tilted objects. In TrueDOP this is only indirectly possible over the length of the presentated shadows. As already mentioned before it should be noticed that the degree of the tilting due to the central perspective presentation and object distance from the principal point in the individual image is random.

In particular objects like wind turbines or power poles, whose structural elements are close to the size of the geometric resolution of the initial DSM, are displayed incomplete. These effect, which influences the DSM as well as the TrueDOP, has to be made familiar to the users. 


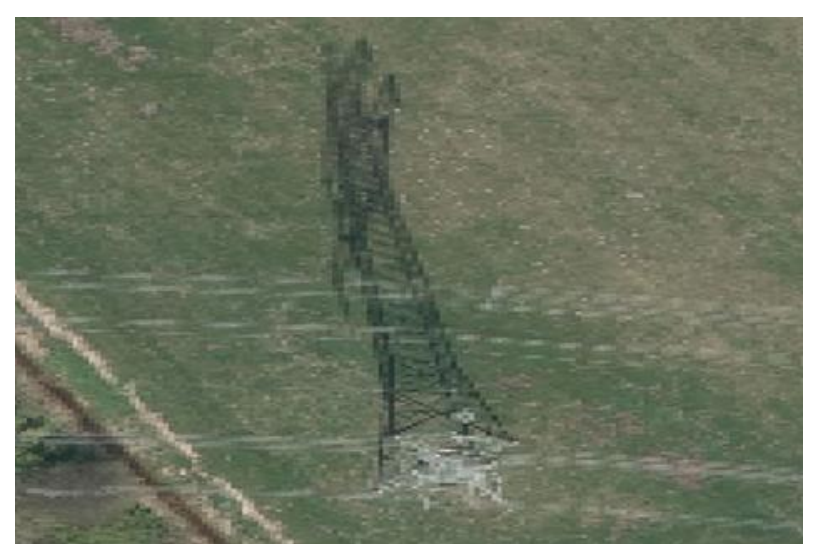

Figure 19. TrueDOP presentation of an electricity pylon

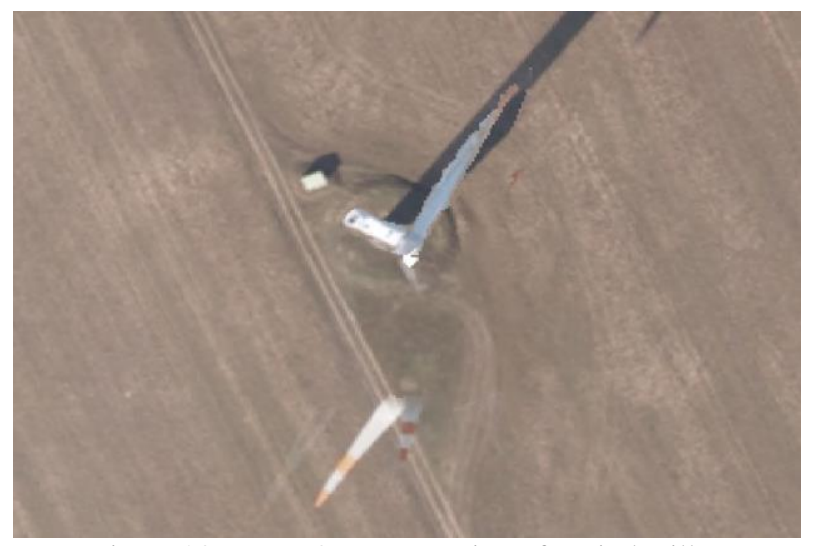

Figure 20. TrueDOP presentation of a wind millXX

2.5.2 Interactive effort shifted in the DSM-quality analysis: The advantages proved the reduce of interactive steps in the TrueDOP-processing. For example, Bridge corrections Seamlines and DTM corrections are dropped. But the effort for the image-based DSM quality analysis is not to be neglected, so that human ressources have to shift in the interactive quality assurance (error analysis and correction) of image-based DSM. This effort, as already mentioned before, produces parallel appreciation of all 3D geodata products, so that it is justifiable.

2.5.3 Ghosting effects of mobile objects: Mobile objects such as driving cars or trains, which have moved during the individual captured aerial images, are mapped transparently in TrueDOP (ghosting effect).

2.5.4 Remaining fringes at object-edges due to geometric and radiometric shadow: At the edges of objects with strong height differences such as buildings data gaps may still be left in the DSM, caused by occlusion and/or shadows. Geometric and radiometric interpolation as well as edge improvements in areas, where no pixel sharp information or only "noisy" information by shadows is available, lead to remaining defects at the edges of objects. These become visible in the image data by using a strong zoom.

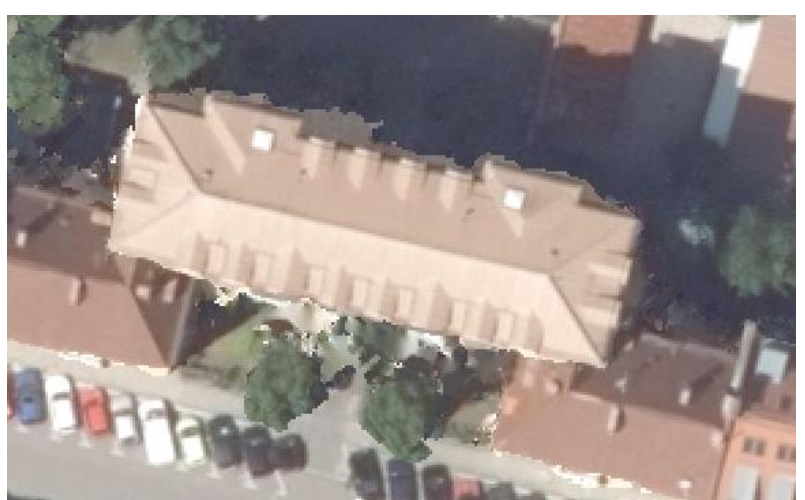

Figure 21. TrueDOP presentation of a building object with

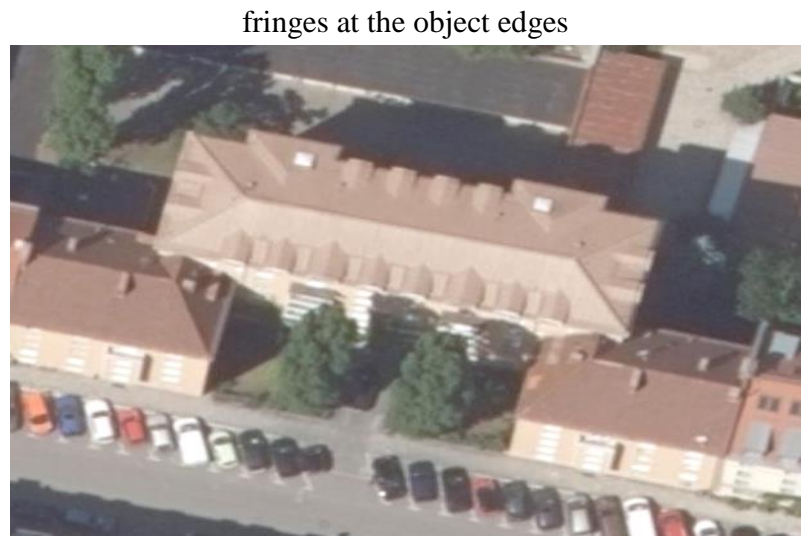

Figure 22. ATKIS-DOP presentation with tilting

\section{CONCLUSIONS}

The German NMA are responsible for basic geodata sets. One standard product, which is well distributed, is the ATKIS-DOP. The improving developments in deriving image-based surface models have made the production of TrueDOP effective and efficient. The experiences in the AdV demonstrate that the most aerial survey projects already fulfill the requirements for derivation of qualitive TrueDOP.

The hardware environment in the NMA have to be adjusted. Some NMA have to invest in additional software licenses for deriving the complete capture area in a sufficient time.

The comparison between TrueDOP and classical ATKIS-DOP delivers more advantages than disadvantages for the decision to use TrueDOP in future. Both increasing quality and reducing human interaction steps are good reasons for TrueDOP introduction. Researches in the quality analysis of image-based surface models have to be enhanced.

Further points, which have to be analysed and discussed in front of the implementation of TrueDOP are:

- Computing Time as a function of hardware environment

- Statements about aerial survey- and data-storage costs

- Costs for the image-based DSM quality management

- $\quad$ customer feedback for new product quality

\section{REFERENCES}

AdV, PG ATKIS-DOP, 2016. Experience report and basis for discussion: Introducing TrueDOP in ATKIS product-chain. (internal paper).

AdV, Productstandard for digital orthophotos (ATKIS®-DOP), www.adv-online.de 